\title{
Reproductive character displacement and underlying drivers in a species-rich and florally diverse lineage of tropical angiosperms (Ruellia; Acanthaceae)
}

\author{
Erin Tripp ${ }^{1}$, Kyle G. Dexter ${ }^{2}$, and Heather Stone ${ }^{3}$ \\ ${ }^{1}$ University of Colorado \\ ${ }^{2}$ The University of Edinburgh \\ ${ }^{3}$ University of Colorado at Boulder
}

May 5, 2020

\begin{abstract}
Reproductive character displacement is a pattern whereby sympatric lineages diverge more in reproductive character morphology than allopatric lineages. This pattern has been observed in many plant species, but comparably few have sought to disentangle underlying mechanisms. Here, in a hyperdiverse lineage of Neotropical plants (Ruellia; Acanthaceae), we present evidence of reproductive character displacement in a macroevolutionary framework (i.e., among species) and document mechanistic underpinnings. In a series of inter-specific hand pollinations in a controlled glasshouse environment, we found that crosses between species that differed more in overall flower size, particularly in style length, were significantly less likely to produce viable seeds. Further, species pairs that failed to set seed were more likely to have sympatric distributions in nature. While these findings could result from competition for pollinators or differential fusion of sympatric populations based on variable crossability, our results instead lend support for a role of reinforcement whereby selection has acted to increase reproductive barriers between sympatric species, especially given divergence in floral traits less likely to be under selection by pollinators (i.e., style length). Our results add to growing evidence that character displacement contributes to exceptional floral diversity of angiosperms.
\end{abstract}

\section{Keywords}

character displacement, experimental, latitudinal gradient, macroevolutionary, pollinator competition, reinforcement, speciation, sympatry

\section{Introduction}

"Endless forms most beautiful" have motivated biologists for centuries (Darwin 1859; Carol 2005), and the remarkable floral diversity of angiosperms is one prime example. Floral diversification in many angiosperm clades is linked to interactions with animal pollinators, given that most angiosperms $(\sim 88 \%)$ are animal pollinated - a number that rises to $94 \%$ within tropical plant communities (Ollerton et al. 2011). Pollinators have behavioral preferences for different rewards, forms, and colors of flowers, which has contributed to a remarkable range of floral diversity (Sargent 2004; Waser and Ollerton 2006; Chittka and Raine 2006; Tripp and Manos 2008; Johnson 2010; Dudash et al. 2011; Van der Niet \& Johnson 2012; Gervasi and Schiestl 2017; Smith and Kriebel 2017). When close relatives within a lineage occur in sympatry and are adapted to 
similar functional groups of pollinators, pollinator competition can arise and negatively impact fitness of one or both plant species (Caruso 2000; Grossenbacher and Stanton 2014; Muchhala et al. 2014; Sletvold et al. 2016). In such instances, selection for floral divergence in sympatry can arise, which has been documented in numerous groups of flowering plants, especially in temperate angiosperms (Sletvold et al. 2016). Pollinator competition can thus lead to greater floral divergence in sympatry compared to allopatry, or reproductive character displacement (RCD; Grossenbacher and Stanton 2014), which represents an important mode of ecological character displacement sensu the classical definition (MacArthur and Levins 1967).

Other mechanisms in addition to pollinator competition can lead to reproductive character displacement, although only some can be attributed to direct selection for character displacement. One such mechanismreinforcement - results from direct selection to reduce gene flow between sympatric, diverging (or already divergent) lineages (Wallace 1889; Coyne and Orr 1989; Matute 2010; Hudson and Price 2014; Hopkins and Rausher 2012). Reinforcement, which has been documented in a limited number of plant lineages, these primarily in temperate regions, describes the process whereby previously allopatric lineages experience selection to avoid costly hybridization after coming into sympatry. In angiosperms, reinforcing selection often operates on floral morphology, thus driving the evolution of morphological divergence in floral traits (Grant 1966; Moyle et al. 2004; Silvertown et al. 2005; Kay and Schemske 2008; Hopkins and Rausher 2012). The underlying assumption of reinforcement is that hybridization is costly because it fails to yield offspring, or offspring have reduced fitness compared to non-hybrid offspring. Reinforcement is often thought to 'complete' the speciation process that begins when populations of species become isolated in allopatry but then later come into contact. While many classic studies of Drosphila and other animals support the concept of reinforcement, it has remained more controversial and less well-documented in plant evolutionary biology (reviewed in Hopkins 2013). Reinforcing selection, if common, is thought to act quickly such that natural hybrids are rarely observed.

Distinguishing between pollinator competition and reinforcement as primary drivers for RCD remains difficult despite the importance of understanding mechanisms that drive plant species divergence and floral diversification. In this study, we propose a two-step approach to help distinguish between these two processes, and then apply this approach to understand floral divergence in sympatry in a species-rich lineage of Neotropical angiosperms (Ruellia L.: Wild Petunias; Fig. 1). The first step involves emphasis on the floral characters themselves that underlie RCD. Pollinators typically select flowers based on visual and olfactory cues that signal reward (nectar and pollen, primarily) and thus divergence in these and associated characters, i.e., color, tube length, and tube width, which frequently co-vary with reward, may signal pollinator competition (Ornelas et al. 2007; Benetiz-Vieyra et al. 2014; Knauer and Schiestl 2014). In contrast, under reinforcement, selection may include traits related to pollinator preference, as above, but is likely to involve additional mechanical forms of isolation or structural incompatibilities that prevent cross fertilization (Kay and Schemske 2008; Hopkins 2013). Thus, divergence in other traits not typically associated with pollinator preference, such as style length or pollen tube length, lends support to hypotheses of reinforcement over pollinator competition.

As a second step, artificial cross pollinations and resultant data on reproductive incompatibility (RI) can be employed to help further distinguish pollinator competition from reinforcement. Under pollinator competition alone as the primary driver for RCD, selection should act to reduce visitation of a given pollinator to different plant lineages (species or incipient species), but other mechanisms to prevent hybridization such as mechanical or intrinsic isolating factors are not expected to manifest between plant lineages. In contrast, under reinforcement, plant lineages divergent in floral morphology should be recalcitrant to artificial hybridization because of mechanical incompatibilities that arise to prevent further, maladaptive hybridization. Hand pollinations bypass the action of pollinators and therefore offer additional means to distinguish between reinforcement and competition for pollinators. If hand pollinations between lineages with dissimilar flowers consistently yield non-viable offspring, reinforcement may be a primary driver of RCD. In contrast, if such hand pollinations do consistently yield viable offspring, then pollinator competition may instead be a primary driver of RCD. 
In this study, we examine a species-rich and florally diverse lineage of tropical angiosperms to (1) test for RCD between species pairs and then (2) evaluate evidence in support of two different mechanisms that contribute to RCD: pollinator competition and reinforcement. We first determine which characters show the strongest pattern of RCD between species pairs. Second, we use hand pollinations in a carefully controlled glasshouse environment to test whether floral dissimilarity is correlated with RI. Finally, we assess if species pairs show greater post-pollination RI when in sympatry compared to allopatry by incorporating geographical range overlap as well as other potential effects, specifically phylogenetic relatedness. Finding that dissimilarity in floral traits (that are unlikely selected for by pollinators) is correlated with RI and that sympatric species cannot produce viable offspring is here taken as evidence in support of reinforcement, whereas finding that floral dissimilarity is unrelated to RI is taken as evidence in support of pollinator competition. The results from this study have implications for understanding the relative contribution of RCD to floral diversification, especially given few examples are known from the tropics (but see Kay and Schemske 2008; Muchhala et al. 2014), and serve as steps towards disentangling the underlying drivers of RCD.

\section{Materials and Methods}

To determine whether crossing success was impacted by floral similarity, we quantified floral used in subsequent crossing trials. We genereated data from five flowers per species for the following traits: length and width of the corolla tube, throat and lobes, peduncle thickness, style length, and ovary length. To determine whether crossing success was influenced by vegetative similarity (vs. floral similarity, above) we additionally quantified vegetative phenotypic divergence for these species based on five leaves per species for the following traits: leaf length, length width, petiole length, number of secondary veins, leaf apex angle and leaf base angle. We used an Ocean Optics JAZ Spectrometer to assess floral color differences following McCarthy et al. (2017). Floral reflectance was measured three times per representative corolla at a 45@ angle. Resulting curves were averaged and then compared across species. Overlapping spectra suggested five clear floral color bins based on curve shape, reflectance wavelength, and median peak height: purple, red, pink, yellow/green, or white (Supplementary Appendix).

To quantify the potential for hybridization, we attempted inter-specific crosses for 16 species of Ruellia (Fig. 2) growing in controlled environment glasshouses at University of Colorado. These species were selected because they derived from the full geographical Neotropical range of Ruellia, with some occurring regularly in sympatry and others not. Because not all species flower at the same time, we were able to attempt crosses between a total of 33 pairs of species, in both directions. We focus on these pairwise comparisons when estimating drivers of crossing success, including floral similarity and geographical range overlap.

Hand pollinations. Hand pollinations were conducted on fresh, fully anthetic flowers by brushing mature, pollen-coated anthers against receptive stigmas (protocol adapted from Long 1966). This approach mirrors the direct transfer of pollen by animal pollinators in natural environments, which characterizes all species of Ruellia . Prior to pollinations, pollen grains were assessed visually under 10x handlens magnification for maturity, which is correlated to anther dehiscence inRuellia. To ensure pollen grain viability, one of the four anthers produced by each species was removed and inspected using the lactophenol-aniline blue stain protocol (Maneval 1936). Stigmas were assumed to be receptive at the time of pollen maturity. For each cross, we mimicked normal pollen load by estimating the average mass produced by anthers of the maternal plant and then adjusting the dosage of pollen donated by the paternal plant accordingly. All crosses consisted of 100\% interspecific pollen. Pollinations were conducted between 09:00-17:00. Immediately following hand pollination, receptive flowers were marked using a colored thread system to track multiple crosses on a single individual. Threads were tied loosely but securely around floral peduncles. A small pilot study conducted on flowers and leaves of six species prior to implementation of the above tracking method indicated that loose threading neither caused nor hastened tissue senescence over a two-week period. Following visual inspection of seeds resulting from successful crosses, one to several seeds per fruit were germinated to further confirm cross success. We additionally attempted to germinate seeds from crosses deemed to be unsuccessful based on visual assessment, and none germinated.

All crosses were conducted carefully in a controlled environment in a manner that emulates direct pollen 
transfer by animal pollinators. Crosses were conducted reciprocally, alternating the donor/recipient status in each cross ( $\mathrm{n}=66$ combinations in total for 33 species pairs). The total number of attempted crosses for each combination varied from 2 to 50 , with $88 \%$ of all species pair combinations being attempted at least 10 times. Crosses were monitored daily until they were determined to either fail or succeed. Crosses that failed to form fruits were treated as failed crosses. Crosses that formed fruits but yielded immature and/or non-viable seeds indicate embryo failure and were treated as failed crosses. Fruits that yielded one or more mature, viable seeds based on visual inspection followed by subsequent germination trials were treated as successful crosses.

Molecular Methods. To account for potential effects of genetic (i.e., phylogenetic) distances between species pairs, we employed the matrix from Tripp and McDade (2014a), which was constructed using three chloroplast markers plus the nuclear ITS+5.8S. We pruned this matrix to contain only taxa relevant to the present study (Fig. 2). The new matrix was aligned using PhyDE (Müller et al. 2016) then analyzed using maximum likelihood imlemented in RAxML v8.2 (Stamatakis 2008). We then constructed a temporally calibrated molecular phylogeny using BEAST v1.82 (Drummond et al. 2012), with three fossil constraints (Supplementary Table 1) derived from Tripp and McDade (2014b), to assess temporal divergence between species pairs. Divergence time estimation methods followed Tripp and McDade (2014b).

Statistical Analyses. To formally test for reproductive character displacement in sympatric species pairs, we used a modified ANOSIM (analysis of similarities) approach (Clark 1993). First, following Coyne and Orr (1989) and Moyle et al. (2004), we classified a given species pair as sympatric if the two species overlap in some portion of their ranges. Co-occurrence was determined through collection notes and localities of herbarium specimens and the extensive field data generated by the first author and taxonomic expert on the genus. We quantified overall reproductive character similarity as the mean Euclidean distance between species in a multivariate decomposition of floral trait space, derived from a principal component analysis of the correlation matrix of the nine quantified floral characters. We also quantified the Euclidean distance between species for each individual floral character. Measures of Euclidean distance (or difference) between species for overall leaf form and individual leaf characters were calculated in the same way.

Our modified ANOSIM approach consists of ranking in decreasing order the Euclidean distances between all species pairs for a given character and then calculating how different are sympatric species pairs, compared to allopatric species pairs, for mean observed ranks. Specifically:

$$
R_{\mathrm{anosim}}=\frac{r_{s}-r_{a}}{\frac{n *(n-1)}{4}}
$$

Where $r_{s}$ equals the mean rank of distances between sympatric species and $r_{a}$ equals the mean rank of distances between allopatric species. The $R_{\text {anosim }}$ statistic varies from 1 to -1 . Values of 0 would indicate that allopatric and sympatric species pairs are no more different from each other than expected by chance. A value of 1 would indicate that sympatric species pairs are always more different in floral form for a given floral character than allopatric species pairs, while a value of -1 would indicate that allopatric species pairs are always more different. To assess whether these differences between sympatric and allopatric species pairs are significantly greater than expected by chance, we used a permutation approach where we shuffled the rows and columns of the dissimilarity matrix for a given character and obtained null expectations for the $\mathrm{R}$ value, given the pairwise values being considered (mimicking the same matrix permutation used in standard ANOSIM). This controls for non-independence of data points involving the same species when assessing significance. For a one-tailed test of the hypothesis that sympatric species pairs will diverge significantly more for a given trait than allopatric species pairs, we determined if the observed $\mathrm{R}$ statistic was greater than that in $95 \%$ of the permutations.

To test if sympatric species pairs are more likely to differ in flower color than allopatric species pairs, we conducted an initial chi-squared analysis to assay whether these two categories of species pairs (sympatric vs. allopatric) had different ratios of species pairs with the same versus different flower colors. As this initial test 
showed no difference $\left(\mathrm{X}^{2}=0.01, \mathrm{p}=1\right)$, we did not pursue additional analyses that would have controlled for non-independence of data points.

In order to assess drivers of inter-specific crossing success, we used a generalized linear mixed model (GLMM) framework to assess how geographical range overlap and/or similarity in floral shape and color and similarity in leaf shape impacted the success of interspecific crosses. The response variable was the binomially distributed number of successes and failures for each attempted cross. We included donor and recipient species identities as random effects to control for non-independence of crosses involving the same species and because both donor and recipient species identities have significant effects on crossing success (likelihood ratio tests of binomial GLM with species identify as fixed effect versus null model, for donor: $\mathrm{X}^{2}=71.2, \mathrm{p}<0.001$; and recipient: $\left.\mathrm{X}^{2}=95.2, \mathrm{p}<0.001\right)$. There was no relationship between crossing success in one direction versus the other (Supplementary Fig. $1 ; \mathrm{r}=0.06, \mathrm{p}=0.751$ ), and including individual species pairs as a random effect did not improve our statistical models or change estimates of fixed effects. We also included the genetic distance between species as a fixed effect in analyses to control for this additional potential driver of crossing success. We first compared the performance of models with a single fixed effect (and the random effects) to models with only random effects using likelihood ratio tests. We then constructed a full model with all fixed and random effects and compared this full model to sub-models where each fixed effect was dropped in turn, again using likelihood ratio tests. The formula for the full model is:

$$
\begin{aligned}
& \text { bin(number of crosses, probability of success) } \sim \text { flower colour similarity }+ \text { floral shape similarity } \\
& + \text { leaf shape similarity }+ \text { genetic distance }+ \text { allopatry vs. sympatry }+(1 \mid \text { Recipient Species } \\
& \text { Identity })+(1 \mid \text { Donor Species Identity })
\end{aligned}
$$

We tested for model overdispersion using a chi-squared test with the residual deviance and degrees of freedom. We did not attempt to test for interactions between our fixed effects due to limited sample size.

While our statistical approach accounts for non-independence of data points due to the same species being used in multiple crosses and to variation in phylogenetic relatedness of species (following Tobias et al. 2014), and while also correctly modeling our binomially distributed crossing success data, it is not identical to 'phylogenetically corrected' approaches used in previous studies that tested the effect of sympatry vs. allopatry on reproductive isolation. In order to ensure comparability with previous studies, we conducted an additional statistical test following procedures used by Coyne and Orr (1989) and Moyle et al. (2004). Specifically, we averaged the proportion of successful crosses for all pairs of species that span a given node in our phylogeny to yield a single estimate of crossing success for each node in the phylogeny. Four of the nodes in our phylogeny were not spanned by any species pair in our study and were omitted from further analysis. Seven nodes in the phylogeny have only allopatric species pairs spanning them, while four nodes have sympatric species spanning them. We compared the mean crossing success values for nodes with only allopatric species pairs to that for nodes spanned by sympatric species pairs using a one-tailed non-parametric Wilcoxon test.

\section{Results}

Our results show reproductive character displacement (RCD) between sympatric species of Ruellia relative to allopatric species (Fig. 3). Overall floral form is significantly more different between sympatric species pairs than between allopatric species pairs $\left(R_{\text {anosim }}=0.63, \mathrm{p}=0.017\right)$. Sympatric species were not significantly more different for overall leaf form $\left(R_{\text {anosim }}=0.38, \mathrm{p}=0.121\right)$. When examining individual floral characters, four of these showed a significantly greater difference between sympatric species pairs (Fig. 3), with style length showing the most pronounced difference (Fig. $3 ; R_{\text {anosim }}=0.85, \mathrm{p}<0.001$ ). In contrast, only one leaf character appears to show significantly greater differences between sympatric species pairs relative to allopatric species pairs (leaf length; $R_{\text {anosim }}=0.54, \mathrm{p}=0.036$ ). If conservative Bonferroni corrections are applied to these multiple tests of significance, only style length shows a significantly greater difference between sympatric species pairs relative to allopatric species pairs.

Among 95 total crossing attempts across five sympatric species pairs, only one instance was successful and yielded mature, viable seeds (a single cross between Ruellia conzattii and $R$. hirsuto-glandulosa). In contrast, 
63 of 730 crossing attempts between allopatric species pairs yielded mature viable seeds (Supplementary Table 2). Statistical analyses using generalized linear mixed models to control for the non-independence of data points involving the same species (following Tobias et al. 2014) showed that occurrence in sympatry significantly reduced crossing success of species pairs (Table 1 ; likelihood ratio-test, $\mathrm{X}^{2}=5.00$, d.f. $=1, \mathrm{p}=$ 0.025). This result is supported by a non-parametric Wilcoxon test on a phylogenetically corrected dataset $(\mathrm{W}=6, \mathrm{p}=0.032)$.

Using the same generalized linear mixed model approach, we found that pairs of species with similarly shaped and similarly colored flowers had significantly higher crossing success (Table 1; Fig. 4; flower color: $\mathrm{X}^{2}=$ 18.94, d.f. $=1, \mathrm{p}<0.001$; flower shape: $\mathrm{X}^{2}=5.76$, d.f. $=1, \mathrm{p}=0.016$; multivariate depiction of floral morphospace provided in Supplementary Fig. 2). Meanwhile, similarity in vegetative morphology did not significantly influence crossing success (Table $1 ; \mathrm{X}^{2}=2.91$, d.f. $=1, \mathrm{p}=0.089$ ). We also found that more distantly related species pairs, quantified as interspecific genetic distance in a maximum likelihood phylogeny, had significantly lower crossing success (Table 1 ; Fig. $4 ; \mathrm{X}^{2}=4.29$, d.f. $=1, \mathrm{p}=0.038$ ). Similar results with respect to significance of fixed effects were obtained when testing the effect of time since divergence in a temporally calibrated phylogeny (Supplementary Table 3). In a multivariate analysis of drivers of crossing success across all species pairs, we found the same direction for our fixed effects as in univariate analyses, but floral shape similarity and geographical range overlap of species pairs did not significantly modulate crossing success (Table 1). An assessment of our model showed these two fixed effects to be highly correlated $(\mathrm{r}=0.37)$ whereas none of the other fixed effects were highly correlated with each other $(\mathrm{r}<0.17)$. If either geographical range overlap of species pairs or similarity in floral shape was removed from the model, the model performed better in explaining crossing success (Table 1; $\Delta$ AICc after removing geography $=$ 1.3, $\Delta$ AICc after removing floral shape similarity $=0.9)$, but if both were removed, the model performed worse $(\triangle \mathrm{AICc}=-2.5)$. This may be expected given the documented reproductive character displacement in sympatric species pairs.

\section{Discussion}

The contribution of RCD to Floral Diversity. In plants, a pattern of increased floral divergence among sympatric compared to allopatric populations of species is well-documented (Whalen 1978; Armbruster et al. 1994; Fishman and Wyatt 1999; Coyne and Orr 2004; Muchhala and Potts 2007; Norton et al. 2015; Gögler et al. 2015; Koski and Ashman 2016; Lagomarsino and Muchhala 2019). In this study, we document clear patterns of reproductive character displacement (RCD) in sympatric species of a diverse lineage of Neotropical plants. This adds to a growing body of evidence for pervasiveness of the pattern in plant communities (Grossenbacher and Whittall 2014; Grossenbacher and Stanton 2014; Norton et al. 2015; Koski and Ashman 2016), extending significance of the phenomenon documented in tropical flowering plants. Our data in tandem with results from numerous prior studies suggests that RCD may be a major factor underlying the great floral diversity of angiosperms. The import of RCD is likely intensified among tropical latitudes given the density and diversity of plant-pollinator interactions.

Evidence in Support of Reinforcement . Two principle mechanisms help explain patterns of RCD: pollinator competition (Muchhala et al. 2014) and reinforcement (Kay and Schemske 2008; Hopkins 2013). Character displacement of floral traits can, specifically, arise from competition for pollinators in sympatry between species that are already reproductively isolated (Muchhala et al. 2014). However, our assessment of which flower characters diverge more between sympatric species pairs than allopatric species pairs combined with results from our artificial crossing experiments suggest a role for reinforcement in Ruellia. Using hand pollinations in a controlled glasshouse environment, we found exceptionally high prezygotic isolation between sympatric species pairs: only one of 95 crossing attempts across five sympatric species pairs produced any viable seeds. In contrast, crosses between allopatric species pairs $(n=28$ pairs) were $8 x$ more likely to be successful $(9 \%$ success rate over all crosses; $30 \%$ of crossed allopatric species pairs were successful at least once). We assessed how similar crossed species pairs were in the shape and color of their flowers, and our results suggest that divergence in floral shape, especially style length, may be one underlying mechanism that results in strong barriers to reproductive compatibility between sympatric species. Both results - strong reduction in 
crossability between sympatric compared to allopatric species pairs and divergence in morphological features not likely under selection by pollinators - better fit a model of reinforcement over pollinator competition. In this study, we also found that species with differently colored flowers also have greatly reduced crossing success, but sympatric species pairs were no more likely to have similar or differently colored flowers than allopatric species pairs. It may be that reinforcing selection acts more strongly on style length than it does on flower color.

Pollinators likely select flowers based on their overall reward, shape, and color. In contrast, it is less likely that they select flowers based primarily on style length. Yet, we have shown that style length diverges more between sympatric species pairs relative to allopatric species pairs than any other floral trait measured (Fig. 3). All sympatric species differ in style length by at least $21.8 \mathrm{~mm}$, while 23 of 28 allopatric species pairs differ in style length by lesser amounts (Fig. 3). If competition for pollinators, absent any involvement of reinforcing processes, were driving patterns recovered in our dataset, we would expect all or most floral traits to show divergence in sympatry instead of the highly variable divergence among characters we recovered (Fig. 3 ). Further, style length is a key character that may underlie potential incompatibilities (Kay and Schemske 2008), and selection on style length would serve to generate a pre-zygotic barrier between species where hybridization is maladaptive. Nonetheless, we recognize that reinforcing selection and pollinator competition are not always mutually exclusive: severe costs of pollen transfer between related species in sympatry may involve both types of processes when reproductive isolation is not fully complete and species boundaries remain semipermeable (Grossenbacher and Stanton 2014; Harrison and Larson 2014; Muchhala et al. 2014).

Two important criteria for reinforcing selection to occur are (i) that hybridization between species pairs is possible such that it can be the subject of selection, and (ii) that hybridization is maladaptive and selected against (Kay and Schemske 2008; Hopkins 2013). We have shown that crosses between 30\% of allopatric species pairs are capable of producing viable seeds. Thus, hybridization is at least possible, as was earlier demonstrated by Long (1975). We do not have experimental evidence for the second criterion, but we note that hybrids inRuellia are rare in natural environments despite extensive geographical range overlap and contemporaneous flowering periods among numerous sets of species, suggesting it is maladaptive to be a hybrid (or that hybridization is otherwise rare to begin with). The first author (E. Tripp) has seen and studied nearly 200 of 300 Neotropical species in their native habitats (www.trippreport.com/ruellia-pages) and in only one instance has a natural hybrid been encountered (i.e., between Ruellia brevifolia and R. puri ; Bolivia, E. Tripp et al. 5971 \& 5977 [COLO Herbarium]; only three putative, additional natural hybrids have been reported by other authors in the literature: Daniel 1990 [Ruellia amoena \& R. foetida]; Ezcurra 1993 [Ruellia brevifolia \& R. longipedunculata; R. brevicaulis \& R. coerulea ]).

Finally, increased floral divergence among sympatric compared to allopatric populations of species is exceptionally well-documented in plants, and reinforcement against maladapted hybrids is likely to account for at least some of these instances. That reinforcing selection may sometimes involve action on only a single locus (Hopkins and Rausher 2012) suggests the potential for relatively simplistic genetic framework underlying contributions of reinforcement to the evolution of RI. Taken together, both the potential frequency and simplicity underlying reinforcement suggest that this type of selection may commonly contribute to completing the process of RI (Liou and Price 1994). However, a paucity of studies that have employed comparative data across clades to investigate RCD and its drivers has precluded understanding of whether such processes help drive lineage diversification and, if so, just how pervasive these processes are (Servedio 2004; Yukilevich 2012; Hopkins 2013). That sympatric species pairs in Ruellia are more reproductively isolated and more divergent in floral morphology than are allopatric pairs provides evidence in support of reinforcement.

Alternatively, if reproductive barriers between species in a given lineage are incomplete, sympatric species that are similar in floral morphology may interbreed due to sharing of pollinators coupled with mechanical and genetic compatibility (Templeton 1981). If gene flow between these diverged yet reproductively compatible lineages is recurrent and prolonged, such lineages may 'fuse', likely with the more fit or otherwise more abundant species in a given environment genetically swamping the less fit, less abundant species (Webb et al. 2011). Meanwhile, sympatric species that are highly dissimilar in floral form may be unable to interbreed 
and maintain distinct evolutionary lineages boundaries. Thus, 'differential fusion' (Templeton 1981) can yield a pattern of RCD similar to that driven by reinforcement. Although we cannot fully rule out differential fusion in this study, under such a model (and in contrast to reinforcement), natural hybrids should be commonly observed in nature between incompletely isolated lineages. However, as discussed above, natural hybrids are exceedingly rare inRuellia. Additionally, extensive evidence now documents reinforcement even in the face of gene flow (Matute 2010; Roda et al. 2017).

Additional Drivers of Variation in Crossing Success . Consistent with studies of model systems in animal speciation biology (Coyne and Orr 2001), we found that crossing success declines with increasing time of evolutionary divergence between species pairs. Whereas it is well established in animals that genetic distance is a significant predictor of interspecific fertility (Coyne and Orr 1989), there has historically been less consensus in plants (Edmands 2002; Moyle et al. 2004). Moyle and colleagues (2004) used comparative data from multiple species to demonstrate that increasing genetic distance strongly decreased crossability in one of the investigated study systems (Silene), but not in the other two lineages they examined. Similarly, using a massive dataset on species crossability in Eucalyptus, Larcombe et al. (2015) found decreased reproductive compatibility with increasing genetic distance. Our and other studies (e.g., Scopece et al. 2007; Moyle et al. 2014; Brandvain et al. 2014) confirm a growing generality of this pattern in plants. The implications of this generality with respect to reinforcement are that reinforcement, as a process important to speciation, should scale with genetic distance. That is, at shallower levels of evolutionary divergence, reinforcement may potentially play a greater role in RI than it does at comparatively deeper evolutionary divergences. This prediction should be tested in a carefully controlled experiment focused on a series of species pairs that vary in their degree of phylogenetic relatedness but otherwise have in common other life history attributes.

Variation in Reproductive Character Displacement Across Clades and Latitudes . In addition to genetic distance, at least four factors should increase opportunities for and thus the potential impacts of both reinforcing selection and pollinator competition in driving floral divergence in sympatry: clade taxon richness, geographically wide-ranging and overlapping species, high densities of individuals within populations, and consequences of gene flow. In Ruellia, hundreds of species span one of the largest latitudinal gradients occupied by any lineage of flowering plants: ca. 80 degrees (i.e., from $~ 43 @ \mathrm{~N}$ near Milwaukee, Wisconsin to $\sim 37 @ \mathrm{~S}$ in central Argentina). Over half of the $\sim 300 \mathrm{New}$ World species have broad geographical ranges (i.e., ranges that extend beyond the borders of a single country). Additionally, there exists widespread co-occurrence of both closely related and more distantly related species in Ruellia, and populations often consist of tens to hundreds of individuals (Tripp 2007; McDade and Tripp 2007; Tripp 2010; Tripp and Luján 2017). Species-rich lineages in which close relatives commonly encounter one another in natural environments should, on the whole, witness greater opportunity for floral diversification via either reinforcing selection or pollinator competition. If these opportunities involve maladaptive gene flow, then reinforcement is expected to be strong and fast acting to reduce energetic costs of producing unfit hybrids. If the above predictors are accurate, emergent properties associated with lineages such as total species number, degree of range overlap, and phylogenetic relatedness of co-occurring species should help predict the relative frequency and importance of underlying drivers of reproductive character displacement in natural landscapes.

We expect that these emergent characteristics of lineages associated with opportunity for reinforcing selection and/or pollinator competition should be more pronounced in tropical (compared to temperate) latitudes, where there typically exists much greater taxonomic and functional diversity of pollinators. Thus, variation in phenomena such as reinforcement across latitudes may be one mechanism contributing to latitudinal gradients in sympatric, and perhaps overall, biodiversity.Ruellia and other broadly ranging lineages (e.g.,Asclepias ) provide excellent systems in which to study whether and how processes including reinforcement and, presumably, competition vary with latitude in plants.

\section{Literature Cited}

Armbruster, W. S., M. E. Edwards, and E. M. Debeved. 1994. Floral character displacement generates assemblage structure of western Australian triggerplants (Stylidium ). Ecology 75:315-329. 
Benitez-Vieyra, S., J. Fornoni, J. Pérez-Alquicira, K. Boege, and C. A. Domínguez. 2014. The evolution of signal-reward correlations in bee- and hummingbird-pollinated species of Salvia . Proc R Soc B 281:20132934.

Butlin, R. 1987. Speciation by reinforcement. TREE 2:8-13.

Brandvain, Y., A. M. Kenney, L. Flagel, G. Coop, and A. L. Sweigart. 2014. Speciation and introgression between Mimulus nasutus and Mimulus guttatus. PLoS Genetics 10:e1004410.

Carroll, S. B. 2005. Endless Forms Most Beautiful. WW Norton \& Company Inc., New York, NY. 331 pp.

Caruso, C. M. 2000. Competition for pollination influences selection on floral traits of Ipomopsis aggregata . Evolution 54:1546-1557.

Chittka, L. and N. E. Raine. 2006. Recognition of flowers by pollinators. Curr Opin Pl Biol 9:428-435.

Clarke, K. R. 1993. Non-parametric multivariate analysis of changes in community structure. Australian Journal of Ecology 18:117-143.

Coyne, J. A. and H. A. Orr. 1989. Patterns of speciation inDrosophila . Evolution 43:362-381.

Coyne, J. A. and H. A. Orr. 1997. "Patterns of speciation inDrosophila " revisited. Evolution 51:295-303.

Coyne, J. A., and H. A. Orr. 2004. Speciation. Sinauer Associates, Sunderland, MA. 545 pp.

Daniel, T. F. 1990. New, reconsidered, and little-known Mexican species of Ruellia . Contrib Univ Mich Herb 17:139-162.

Darwin, C. 1859. On the Origin of Species by Means of Natural Selection, or the Preservation of Favoured Races in the Struggle of Life. John Murray Co., London, UK.

Dettman, J. R., J. B. Anderson, and L. M. Kohn. 2008. Divergent adaptation promotes reproductive isolation among experimental populations of the filamentous fungus Neurospora. BMC Evol Biol 8:35.

Dobzhansky, T. 1937. Genetics and the Origin of Species . Columbia University Press, New York.

Drummond, A. J., M. A. Suchard, D. Xie, and A. Rambaut. 2012. Bayesian phylogenetics with BEAUTi and the BEAST 1.7. Mol Biol Evol 29:1969-1973.

Dudash, M. R., C. Hassler, P. M. Stevens, and C. B. Fenster. 2011. Experimental floral and inflorescence trait manipulations affect pollinator preference and function in a hummingbird-pollinated plant. Am J Bot 98:275-282.

Ezcurra, C. 1993. Systematics of Ruellia (Acanthaceae) in southern South America. Ann Mo Bot Gard 80:787-845.

Fishman, L. and R. Wyatt. 1999. Pollinator-mediated competition, reproductive character displacement, and the evolution of selfing inArenaria uniflora (Caryophyllaceae). Evolution 53:1723-1733.

Gavrilets, S. 1997. Hybrid zones with Dobzhansky-type epistatic selection. Evolution 51:1027-1035.

Gervasi, D. D.L. and F. P. Schiestl. 2017. Real-time divergent evolution in plants driven by pollinators. Nat Comm 8:14691.

Grossenbacher, D. L. and J. B. Whittall. 2011. Increased floral divergence in sympatric monkeyflowers. Evolution 65:2712-2718.

Grossenbacher, D. L. and J. B. Whittall. 2014. Pollinator-mediated competiton influences selection for flowercolor displacement in sympatric monkeyflowers. Am J Bot 101:1915-1924.

Grant, V. 1965. Evidence for the selective origin of incompatibility barriers in the leafy-stemmed Gilias. Proc Nat Acad Sci USA 54:1567-1571. 
Grossenbacher, D. L. and M. L. Stanton. 2014. Pollinator-mediated competition influences selection for flower-color displacement in sympatric monkeyflowers. Am J Bot 101:1915-1924.

Gögler, J., J. Stökl, P. Cortis, H. Beyrle, M. R. B. Lumaga, S. Cozzolino, and M. Ayasse. 2015. Increased divergence in floral morphology strongly reduces gene flow in sympatric sexually deceptive orchids with the same pollinator. Evol Ecol 29:703-717.

Harrison, R. G. and E. L. Larson. 2014. Hybridization, introgression, and the nature of species boundaries. J Hered 105:795-809.

Hopkins, R. 2013 Reinforcement in plants. New Phytol 197:1095-1103.

Hopkins, R. and M. D. Rausher. 2011. Identification of two genes causing reinforcement in the Texas wildflower Phlox drummondii. Nature 469:411-415.

Hopkins, R. and M. D. Rausher. 2012. Pollinator-mediated selection on flower color allele drives reinforcement. Science 335:1090-1092.

Hudson, E. J. and T. D. Price. 2014. Pervasive reinforcement and the role of sexual selection in biological speciation. J Hered 105:821-833.

Johnson, S. D. 2010. The pollination niche and its role in the diversification and maintenance of the southern African flora. Phil Trans Royal Soc B 365:499-516.

Kay, K. M. and D. W. Schemske. 2008. Natural selection reinforces speciation in a radiation of neotropical rainforest plants. Evolution 62:2628-2642.

Knauer, A. C. and F. P. Schiestl. 2014. Bees use honest floral signals as indicators of reward when visiting flowers. Ecol Lett 18:135-143.

Koski, M. H. and T. L. Ashman. 2016. Reproductive character displacement and environmental filtering shape floral variation between sympatric sister taxa. Evolution 70:2616-2622.

Lagomarsino, L. P. and N. Muchhala. 2019. A gradient of pollinator specialiation in three species of Bolivia Centropogon. Am J Bot 106:633-642.

Larcome, M. J., B. Holland, D. A. Steane, R. C. Jones, D. Nicolle, R. E. Vaillancourt, and P. M. Potts. 2015. Patterns of reproductive isolation in Eucalyptus - a phylogenetic perspective. Mol Biol Evol 32:1833-1846.

Lemmon, E. M. and A. R. Lemmon. 2009. Reinforcement in chorus frogs: lifetime fitness estimates including intrinsic natural selection and sexual selection against hybrids. Evolution 64:1748-1761.

Levin, D. A. and H. W. Kerster. 1968. Natural selection for reproductive isolation in Phlox . Evolution 43:679-687.

Liou, L. W. and T. D. Price. 1994. Speciation by reinforcement of premating isolation. Evolution 48:14511459 .

Long, R. W. 1966. The artificial intersectional hybrid of the tropical species Ruellia brittoniana x R. occidentals and its taxonomic significance. Bull Torr Bot Club 93:181-187.

Long, R. W. 1975. Artificial interspecific hybridization in temperate and tropical species of Ruellia (Acanthaceae). Brittonia 27:289-296.

Lowry, D. B., J. L. Modliszewski, K. M. Wright, C. A. Wu, and J. H. Willis. 2008. The strength and genetic basis of reproductive isolating barriers in flowering plants. Phil Trans R Soc B 363:3009-3021.

Maneval, W.E. 1936. Lactophenol preparations. Stain Technol 11:9-11.

Matute, D. R. 2010. Reinforcement can overcome gene flow during speciation in Drosophila . Curr Biol 20:2229-2233. 
McCarthy, E. W., A. E. Berardi, S. D. Smith, and A. Litt. 2017. Related allopolyploids dsiplay distinct floral pigment profiles and transgressive pigments. Am J Bot 104:92-101.

McDade, L. A., and E. A. Tripp. 2007. Synopsis of Costa RicanRuellia L. (Acanthaceae), with descriptions of four new species. Brittonia 59:199-216.

Moyle, L. C., M. S. Olson, and P. Tiffin. 2004. Patterns of reproductive isolation in three angiosperm genera. Evolution 58:1195-1208.

Muchhala, N., S. Johnsen, and S. D. Smith. 2014. Competition for hummingbird pollination shapes flower color variation in Andean Solanaceae. Evolution 68:2275-2286.

Muchhala, N. and M. D. Potts. 2007. Character displacement among bat-pollinated flowers of the genus Burmeistera : analysis of mechanism, process and pattern. Proc Royal Soc B 274:2731-2737.

Müller, K., D. Müller, C. Neinhuis, and D. Quandt. 2016. PhyDE: Phylogenetic Data Editor. Available at: http://www.phyde.de

Nista, P., A. N. Brothers, and L. F. Delph. 2015. Differences in style length confer prezygotic isolation between two dioecious species of Silene in sympatry. Ecol Evol 5:2703-3711.

Noor, M. 1999. Reinforcement and other consequences of sympatry. Heredity 83:503-508.

Norton, N. A., M. T. R. Fernando, C. Herlihy, and J. W. Busch. 2015. Reproductive character displacement shapes a spatially structured petal color polymorphism in Leavenworthia stylosa . Evolution 69:1191-1207.

Nosil, P., and R. Yukilevich. 2008. Mechanisms of reinforcement in natural and simulated polymorphic populations. Biol J Linn Soc 95:305-319.

Nosil, P. and D. Schluter. 2011. The genes underlying the process of speciation. TREE 26:160-167.

Nosil, P. 2012. Degree of sympatry affects reinforcement inDrosophila. Evolution 67:868-872.

Ollerton, J., R. Winfree, and S. Tarrant. 2011. How many flowering plants are pollinated by animals? Oikos 120:321-326.

Ornelas, J. F., M. Ordano, A. J. De-Nova, M. E. Quintero, and T. Garland. 2007. Phylogenetic analysis of interspecific variation in nectar of hummingbird-visited plants. J Evol Biol 20:1904-1917.

Pascarella, J. B. 2007. Mechanisms of prezygotic reproductive isolation between two sympatric species, Gelsemium rankinii and G. sempervirens (Gelsemiaceae), in the southeastern United States. Am J Bot 94:468-576.

Prager, E. M. and A. C. Wilcson. 1975 Slow evolutionary loss of the potential for interspecific hybridization in birds: a manifestation of slow regulatory evolution. Proc Nat Acad Sci USA 72:200-204.

Rabosky, D. L. and D. R. Matute. 2013. Macroevolutionary speciation rates are decoupled from the evolution of intrinsic reproductive isolation in Dropsophila and birds. Proc Nat Acad Sci USA 110:15354-15359.

Rieseberg, L. H., and J. H. Willis. 2007. Plant speciation. Science 317:910-914.

Roda, F., F. K. Mendes, M. W. Hahn, and R. Hopkins. 2017. Genomic evidence of gene flow during reinforcement in Texas Phlox. Mol Ecol 26:2317-2330.

Runquist, R. D. B., and D. A. Moeller. 2014. Floral and mating system divergence in secondary sympatry: testing an alternative hypothesis to reinforcement in Clarkia. Ann Bot 113:223-235.

Runquist, R. B., D. Grossenbacher, S. Porter, K. Kay, and J. Smith. 2016. Pollinator-mediated assemblage processes in California wildflowers. J Evol Biol 29:1045-1058.

Saetre, G. P., T. Moum, S. Bures, M. Král, M. Adamjan, and J. Moreno. 2007. A sexually selected character displacement in flycatchers reinforces premating isolation. Nature 387:589-592. 
Santamour, F. S. 1972. Interspecific hybridization in Platanus . Forest Sci 18:236-239.

Sargent, R. D. 2004. Floral symmetry affects speciation rates in angiosperms. Proc R Soc Lond B 271:603-608.

Scopece, G., A. Musacchio, A. Widmer, and S. Cozzolino. 2007. Patterns of reproductive isolation in Mediterranean deceptive orchids. Evolution 61:2623-2642.

Silvertown, J., C. Servaes, P. Biss, and D. Macleod. 2005. Reinforcement of reproductive isolation between adjacent populations in the Park Grass Experiment. Heredity 95:198-205.

Sletvold, N., J. Trunschke, M. Smit, J. Verbeek, and J. Agren. 2016. Strong pollinator-mediated selection for increased flower brightness and contrast in a deceptive orchid. Evolution 70:716-724.

Sobel, J. M., G. F. Chen, L. R. Watt, and D. W. Schemske. 2009. The biology of speciation. Evolution 64:295-315.

Soltis, D. E., P. S. Soltis, T. S. Collier, and M. L. Edgerton. 1991. Chloroplast DNA variation within and among genera of the Heucheragroup (Saxifragaceae): evidence for chloroplast transfer and paraphyly. Am J Bot 78:1091-1112.

Smith, S. D. and R. K. Kriebel. 2017. Convergent evolution of floral shape tied to pollinator shifts in Iochrominae (Solanaceae). Evolution 72:688-697.

Spriggs, E. L., C. Schlutius, D. A. Eaton, B. Park, P. W. Sweeney, E. J. Edwards, and M. J. Donoghue. 2019. Differences in flowering time matinain species boundaries in a continental radiation of Viburnum . Am J Bot 106:833-849.

Stamatakis, A. 2008. RAxML version 8: a tool for phylogenetic analysis and post-analysis of large phylogenies. Bioinformatics 1:1312-1313.

Templeton, A. R. 1981. Mechanisms of speciation: a population genetic approach. Ann Rev Ecol Syst 12:2348.

Tripp, E. A. 2007. Evolutionary relationships within the species-rich genus Ruellia (Acanthaceae). Syst Bot 32:628-649.

Tripp, E. A. and P. S. Manos. 2008. Is floral specialization an evolutionary dead-end? Pollination system transitions in Ruellia(Acanthaceae). Evolution 62:171-1737.

Tripp, E. A. 2010. Taxonomic revision of Ruellia sect.Chiropterophila (Acanthaceae): a lineage of rare and endemic species from Mexico. Syst Bot 35:629-661.

Tripp, E. A., S. Fatimah, I. Dabryshire, and L. A. McDade. 2013. Origin of African Physacanthus (Acanthaceae) via wide hybridization. PLoS ONE 8:e55677.

Tripp, E. A. and L. A. McDade2014a. Time-calibrated phylogenies of hummingbirds and hummingbirdpollinated plants reject a hypothesis of diffuse co-evolution. Aliso 31:89-103.

Tripp, E. A. and L. A. McDade. 2014b. A rich fossil record yields calibrated phylogeny for Acanthaceae (Lamiales) and evidence for marked biases in timing and directionality of intercontinental disjunctions. Sys Bio 63:660-684.

Tripp, E. A. and M. Luján. 2017. Venezuelan Ruellia: A Monograph.Memoirs of the New York Botanical Garden. 179 p.

Turelli, M., J. R. Lipkowitz, and Y. Brandvain. 2013. On the Coyne and Orr-igin of species: effects of intrinsic postzygotic isolation, ecological differentiation, $\mathrm{X}$ chromosome size, and sympatry onDrosophila speciation. Evolution 68:1176-1187. 
Turner, E., D. J. Jacobson, and J. W. Taylor. 2011. Genetic architecture of a reinforcd, postmating, reproductive isolation barrier betweenNeurospora species indicates evolution via natural selection. PLoS Genetics 7:e1002204.

Van der Niet, T., S. D. Johnson, and H. P. Linder. 2006. Macroevolutionary data suggest a role for reinforcement in pollination system shifts. Evolution 60:1596-1601.

Van der Niet, T. and S. D. Johnson. 2012. Phylogenetic evidence for pollinator-driven diversification of angiosperms. TREE 27:353-361.

Wallace, A. R. 1889. Darwinism . Macmillan \& Co.: London.

Waser, N. M. and J. Ollerton. 2006. Plant-Pollinator Interactions: from Specialization to Generalization . University of Chicago Press, Chicago, IL. 488 pp.

Webb, W. C., and J. M. Marzluff, and K. E. Omland. 2011. Random interbreeding between cryptic lineages of the commen raven: evidence for speciation in reverse. Molecular Ecology 20:2390-2402.

Yardeni, G., N. Tessler, E. Imbert, Y. Sapir. 2016. Reproductive isolation between populations of Iris atropurpurea is associated with ecological differentiation. Annals of Botany 118:971-982.

Yukilevich, R. 2012. Asymmetrical patterns of speciation uniquely support reinforcement in Drosophila . Evolution 66:1430-1446.

Zwickl, D. J. 2006. Genetic algorithm approaches for the phylogenetic analysis of large biological sequence datasets under the maximum likelihood criterion. Ph.D dissertation, The University of Texas at Austin.

Table 1 . Estimates of fixed effects, with standard errors, from univariate models and a multivariate model to explain crossing success in Ruellia (Acanthaceae). A negative coefficient for Allopatry vs. Sympatry indicates reduced crossing success in sympatry relative to allopatry. Genetic distance is measured as the branch length separating two species in a maximum likelihood phylogeny. Asterisks indicate significance levels from likelihood ratio tests that (1) compare the likelihood of a model with just the single fixed effect and random effects for donor and species identity versus a null model with only random or (2) compare the likelihood of the full model with all fixed effects and random effects versus a model without the given fixed effect $\left[\mathrm{p}<0.05^{*}, \mathrm{p}<0.01^{* *}, \mathrm{p}<0.001^{* * *}\right]$.

Univariate model estimate with S.E. Multivariate model estimate with S.E.

\begin{tabular}{lll}
\hline Allopatry vs. Sympatry & $-2.40 \pm 1.32^{*}$ & $-1.63 \pm 1.54$ \\
Genetic Distance & $-36.4 \pm 18.1^{*}$ & $-40.9 \pm 20.2^{*}$ \\
Flower Color Similarity & $2.62 \pm 0.71^{* * *}$ & $2.34 \pm 0.80^{* * *}$ \\
Flower Shape Similarity & $0.64 \pm 0.29^{*}$ & $0.51 \pm 0.41$ \\
Leaf Shape Similarity & $0.35 \pm 0.21$ & $0.05 \pm 0.96$
\end{tabular}

\section{Figure legends}

Figure 1. Morphological diversity of species of Ruelliaused in this study. A. R. breedlovei . B. R. macrantha . C.R. elegans . D. R. lutea . E. R. matudae . F. R. morongii . G. R. californica . H. R. hirsutoglandulosa . I.R. saccata. J. R. speciosa. K. R. longipedunculata .

Figure 2. Experimental design of crossing study, showing that both allopatric and sympatric crosses were attempted within and across major clades of Ruellia. Lines connect species pairs for which crosses were attempted (all crosses attempted bidirectionally). Dashed lines: allopatric species pairs. Solid lines: sympatric species pairs. Circles next to species names colored according to flower color. Circles are proportional to flower size (first axis of principal component analysis in Supplementary Fig. 2) and depict an overall lack of phylogenetic signal for flower color or size. Phylogeny rotated around select nodes for visual clarity. 
Figure 3. Left panel : F-statistic for analyses of variance that compare interspecific distances for a given floral characteristic in sympatric vs. allopatric species pairs. High values indicate that sympatric species pairs diverge more for a given floristic characteristic relative to allopatric species pairs. Low values indicate equivalent divergences. Right panel : raw style length measurements for each species pair for which a cross was attempted; each pair represented by a vertical line and end points depict style lengths for the two species. All sympatric species pairs differ by at least $21.8 \mathrm{~mm}$ in style length, while 23 of 28 allopatric species pairs differ by less than $21.8 \mathrm{~mm}$ in style length.

Figure 4. Impacts of genetic distance, measured as interspecific phylogenetic distance in a maximum likelihood phylogeny (panels A \& C) and flower shape, measured as euclidean distance in a principal components decomposition of floral shape measurements (panels B \& D) on crossing success. Upper panels (A \& B): allopatric species pairs. Lower panels (C \& D): sympatric species pairs. Floral similarity and genetic distance significantly impacted crossing success. Covariance in flower shape and geography evident in panel $\mathrm{D}$ (no sympatric species pairs with a flower shape distance $<3$ ). In $\mathrm{C} \& \mathrm{D}$, note that only one sympatric cross was successful. Points staggered slightly on $\mathrm{x}$-axis for visual clarity.

\section{Hosted file}

Figures.docx available at https://authorea.com/users/292145/articles/419814-reproductivecharacter-displacement-and-underlying-drivers-in-a-species-rich-and-florally-diverse-

lineage-of-tropical-angiosperms-ruellia-acanthaceae 